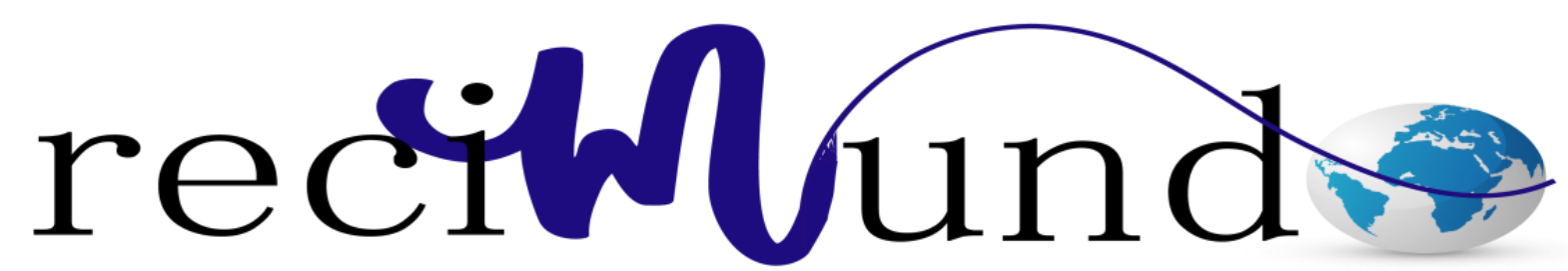

Revista Cientifica Mundo de la lnvestígación y el Conocimiento

Charles David Altamirano Olvera ${ }^{\text {a }}$; Elizabeth Katiuska Villegas Guerrero ${ }^{\text {b }}$; Henrry Willian Sacoto Carranza ${ }^{c}$; Carmen Leonor González Plúa ${ }^{\mathrm{d}}$

Síndrome de desgaste profesional en médicos residentes e internos de medicina del área de emergencia del hospital universitario periodo septiembre 2015 a diciembre 2015

Professional attrition syndrome in medical residents and medical interns in the emergency area of the university hospital period September 2015 to December 2015

Revista Científica Mundo de la Investigación y el Conocimiento. Vol. 3 núm.3. Esp., noviembre, ISSN: 2588-073X, 2019, pp. 962-981

DOI: $10.26820 /$ recimundo/3.(3.Esp).noviembre.2019.962-981

URL: http://recimundo.com/index.php/es/article/view/638

Código UNESCO: 3204.03 Salud Profesional

Tipo de Investigación: Artículo de Revisión

(C) RECIMUNDO; Editorial Saberes del Conocimiento, 2019

Recibido: 15/09/2019

Aceptado: 23/10/2019

Publicado: 30/11/2019

Correspondencia: chardei27_29@ hotmail.com

a. Médico; Investigador Independiente; Guayaquil, Ecuador; chardei27_29@hotmail.com

b. Médico; Investigador Independiente; Guayaquil, Ecuador; kattys 92@ hotmail.es

c. Médico; Investigador Independiente; Guayaquil, Ecuador; henrry20sacoto @ hotmail.com

d. Médico; Investigador independiente; Guayaquil, Ecuador; carmigp25@ gmail.com 


\section{Síndrome de desgaste profesional en médicos residentes e internos de medicina del área de emergencia del hospital universitario periodo septiembre}

2015 a diciembre 2015

Vol. 3, núm. 3 Esp., (2019)

Charles David Altamirano Olvera; Elizabeth Katiuska Villegas Guerrero; Sacoto Carranza Henrry Willian; González Plúa Carmen Leonor

\section{RESUMEN}

El Síndrome de Desgaste Profesional es un trastorno tridimensional de agotamiento emocional, despersonalización y baja realización personal en el trabajo. Aparece de forma específica en las profesiones de ayuda en relación con las interacciones humanas, y se instaura de forma progresiva afectando tanto al profesional como a la organización, actualmente es la causa más frecuente de baja laboral.

En el presente estudio de tipo descriptivo no experimental se pudo conocer el porcentaje de trabajadores de la salud que presentó el síndrome de desgaste profesional el mismo que se realizó en el área de emergencia del Hospital Universitario de Guayaquil el cual está dividido en tres áreas que son: emergencia de adultos, pediátrica y ginecológica en el que laboran 104 individuos de los cuales 72 son internos de medicina de la Universidad de Guayaquil y 32 médicos residentes como herramienta de investigación se aplicó el cuestionario de Maslach Burnout Inventory demostrando que el 16\% del universo cumplió el criterio de puntuaciones altas en las tres dimensiones $\uparrow \mathrm{CE} \uparrow \mathrm{DP} \downarrow \mathrm{RP}$ lo que nos lleva a un $16 \%$ de nuestra muestra de estudio a su vez la edad media de presentación del síndrome de desgaste profesional fue de 26.6 años, con una desviación estándar de 2.44 años, se da con mayor frecuencia en la emergencia de adultos en un $13 \%$, debido al no descanso durante la guardia que se destacó en un $90 \%$, seguido de emergencia ginecológica de un $3 \%$ y no viéndose afectada emergencia pediátrica, el $21 \%$ de los Internos de Medicina en estudio fueron afectados en comparación con el $6 \%$ de Médicos Residentes afectados, el $84 \%$ en estudio no llegó a cumplir el criterio pero presentando algunas de las dimensiones de desgaste profesional que numerosos estudios mencionan que la fluidez de pacientes, espacio reducido para la atención, mayor contacto con la muerte y el dolor de los usuarios hace que se desarrolle más el síndrome.

Palabras Claves: Síndrome de desgaste profesional; Médicos residentes; Internos de medicina; Emergencia. 
Síndrome de desgaste profesional en médicos residentes e internos de medicina del área de emergencia del hospital universitario periodo septiembre 2015 a diciembre 2015

Vol. 3, núm. 3 Esp., (2019)

Charles David Altamirano Olvera; Elizabeth Katiuska Villegas Guerrero; Sacoto Carranza Henrry Willian; González Plúa Carmen Leonor

\section{ABSTRACT}

Professional Burnout Syndrome is a three-dimensional disorder of emotional exhaustion, depersonalization and low personal accomplishment at work. Specifically listed in the helping professions regarding human interactions, and establishes progressively affecting both the professional and the organization, is currently the most frequent cause of absenteeism.

In the present study of non-experimental descriptive could know the percentage of health workers who presented the professional burnout syndrome the same as was done in the emergency area of the University Hospital of Guayaquil which is divided into three areas that are : emergency adult, pediatric and gynecological in which they work 104 individuals of which 72 are internal medicine at the University of Guayaquil and 32 medical residents as a research tool questionnaire Maslach Burnout Inventory was applied showing that $16 \%$ of the universe fulfilled the criterion of high scores in all three dimensions $\uparrow \mathrm{EC} \uparrow \mathrm{DP} \downarrow \mathrm{RP}$ which leads to $16 \%$ of our study sample turn the average age of onset of burnout syndrome was 26.6 years, with a deviation standard 2.44 years, it occurs most frequently in adult emergence by $13 \%$ due to not break during the guard that stood out by $90 \%$, followed by gynecological emergency $3 \%$ and not be affected pediatric emergency, $21 \%$ of Internal Medicine study were affected compared with $6 \%$ of Resident Doctors affected, $84 \%$ in the study failed to meet the criteria but showing some of the dimensions of burnout that numerous studies mention that fluency of patients, reduced space for care, more contact with death and pain of users makes the syndrome is developed further.

KeyWords: Burnout syndrome; Residents Doctors; Internal Medicine; Emergency. 


\section{Síndrome de desgaste profesional en médicos residentes e internos de medicina del área de emergencia del hospital universitario periodo septiembre}

2015 a diciembre 2015

Vol. 3, núm. 3 Esp., (2019)

Charles David Altamirano Olvera; Elizabeth Katiuska Villegas Guerrero; Sacoto Carranza Henrry Willian; González Plúa Carmen Leonor

\section{Introducción.}

La Carta Constitucional de la Organización Mundial de la Salud (OMS, 1948), definió la salud como "El estado completo de bienestar físico, mental y social y no solamente la ausencia de afecciones o enfermedades”. (OMS, 2016)

Afortunadamente con esta definición se mejoró el concepto de salud, ya que dejó de ser la “ausencia de enfermedad” para pasar a ser "bienestar físico, mental y social”. (Galván, Vassallo, Rodríguez, \& Otero, 2014)

Con esta concepción holística de la salud que asume que el individuo busca, no solamente no estar enfermo sino además alcanzar un estado de felicidad y bienestar que está relacionado con otros factores tales como la calidad de vida. De este concepto el estudio del Síndrome de desgaste profesional, conocido también como burnout o "estar quemado", pretende incluir una perspectiva psicosocial en la que se analicen las variables del contexto socioprofesional en el que el médico está inmerso y se analicen las consecuencias del desgaste profesional en su salud. (Quevedo Bastias, 2006) (Ewart, Marcus, Gaba, \& Brandnen, 2004)

Este es un síndrome clínico descrito por primera vez por Freudenberger (1974) como un “estado de fatiga o frustración que se produce por la dedicación a una causa, forma de vida o de relación, que no produce los resultados esperados". Esto nos lleva a un agotamiento emocional, perdida de interés e implicación por una actividad con la que previamente se estuvo comprometido y a la que se dedicó un gran esfuerzo. 


\section{Síndrome de desgaste profesional en médicos residentes e internos de medicina del área de emergencia del hospital universitario periodo septiembre 2015 a diciembre 2015}

Vol. 3, núm. 3 Esp., (2019)

Charles David Altamirano Olvera; Elizabeth Katiuska Villegas Guerrero; Sacoto Carranza Henrry Willian; González Plúa Carmen Leonor

Cristina Maslach (1982) lo define como un síndrome psicológico caracterizado por tres componentes principales cansancio emocional, despersonalización y baja realización personal de quienes trabajan con personas, que surge como respuesta al estrés crónico en el trabajo. (Lopez León, Rodríguez Moctensuma, \& López Carbona, 2007)

El cansancio emocional mide los sentimientos de agotamiento debidos a las relaciones interpersonales, la despersonalización mide la falta de interés a lo que aqueja el paciente y la realización personal mide los sentimientos de competencia y éxitos en el trabajo.

El interés del estudio del desgaste profesional en el ámbito médico es una consecuencia de las crecientes demandas sociales, organizacionales y emocionales que afectan en la actualidad al desarrollo de la profesión y que forman parte de la etiología del síndrome.

Se realizó una amplia búsqueda de datos tanto en bibliotecas de instituciones de nivel superior como de buscadores de referencia global como Google Academics, Pubmed entre otras.

Dado los resultados de estos estudios, esta investigación tiene como propósito conocer el nivel de desgaste profesional que existe en las diferentes áreas de emergencia del Hospital Universitario, a través de la recolección de datos empleando una encuesta previamente validada a Médicos Residentes e Internos de Medicina, utilizando como instrumento la escala de valoración de Maslach Burnout Inventory (MBI) el cual es el instrumento validado más utilizado para evaluar el desgaste profesional en personas que atienden a seres humanos, desde que fue presentado en el año de 1981. (Sos Tena, Sobrequés Soriano, \& Segura Bernal, 2002) 


\section{Síndrome de desgaste profesional en médicos residentes e internos de medicina del área de emergencia del hospital universitario periodo septiembre}

2015 a diciembre 2015

Vol. 3, núm. 3 Esp., (2019)

Charles David Altamirano Olvera; Elizabeth Katiuska Villegas Guerrero; Sacoto Carranza Henrry Willian; González Plúa Carmen Leonor

El objetivo del presente estudio busca determinar los factores de riesgo en el desarrollo del síndrome de desgaste profesional en los Médicos Residentes e Internos de Medicina del área de emergencia del hospital universitario periodo septiembre 2015 a diciembre 2015.

\section{Metodología.}

Lugar de investigación

El estudio se llevará a cabo en el área de Emergencia del Hospital Universitario de Guayaquil ubicado en la parroquia Guayaquil Vía Perimetral Km 23.5, dicha área está conformada por tres secciones: Emergencia de Adultos, Emergencia Pediátrica y Emergencia Ginecológica.

\section{Caracterización de la zona de trabajo}

Guayaquil, conocida como la "Perla del Pacífico", es una ciudad que encierra historia y tradición. Fue fundada en 1538 y, en la época de la Colonia, se convirtió en el astillero más importante de América del Sur. Hoy, Guayaquil, capital económica del Ecuador es el principal puerto del país, considerado como uno de los más importantes del mundo, porque su ubicación e infraestructura brinda facilidades para el comercio marítimo internacional.

Cuenta con 2`350.915 de habitantes, en la zona Urbana 2`278.691 hab. Y la Rural 72.224 hab.

La Universidad de Guayaquil, conocida coloquialmente como la Estatal, es una universidad pública localizada en la ciudad de Guayaquil. Es la universidad más grande del país y la más 


\section{Síndrome de desgaste profesional en médicos residentes e internos de medicina del área de emergencia del hospital universitario periodo septiembre 2015 a diciembre 2015}

Vol. 3, núm. 3 Esp., (2019)

Charles David Altamirano Olvera; Elizabeth Katiuska Villegas Guerrero; Sacoto Carranza Henrry Willian; González Plúa Carmen Leonor

antigua de la ciudad, además de tener seis extensiones universitarias en varias partes del país. Actualmente tiene una población estudiantil que bordea los 80.000 estudiantes, siendo por ello, considerada como universidad con mayor alumnado en todo el país. La universidad está comprendida por 17 facultades que imparten 31 carreras de pregrado, además de cinco institutos de carreras de postgrado. La Facultad de Medicina y específicamente su Escuela de Medicina tiene una población estudiantil de más de 6.000 estudiantes. Se encuentra ubicada en Guayaquil, Ciudadela Universitaria Salvador Allende, Malecón del Salado entre Av. Fortunato Safadi (Av. Delta) y Av. Kennedy.

\section{Periodo de investigación}

El periodo de investigación corresponde a los meses de septiembre de 2015 a diciembre de 2015.

Universo

El universo corresponde a los Internos de Medicina distribuidos en las 4 guardias de las tres Áreas de Emergencias que son los siguientes: Emergencia de Adultos: 28, Emergencia Pediátrica: 24 y Emergencia Ginecológica: 20; y también los Médicos Residentes distribuidos en las 4 guardias de las tres Áreas de Emergencias que son los siguientes: Emergencia de Adultos: 20, Emergencia Pediátrica: 4 y la Emergencia de Ginecológica: 8.

Muestra 


\section{Síndrome de desgaste profesional en médicos residentes e internos de medicina del área de emergencia del hospital universitario periodo septiembre}

2015 a diciembre 2015

Vol. 3, núm. 3 Esp., (2019)

Charles David Altamirano Olvera; Elizabeth Katiuska Villegas Guerrero; Sacoto Carranza Henrry Willian; González Plúa Carmen Leonor

La muestra corresponde a un total de 104 Personas. Los mismos que están comprendidos en 72 Internos de Medicina de la Universidad Estatal y a 32 Médicos Residentes del Área de Emergencia.

\section{Viabilidad}

Esta investigación es viable porque consta con el permiso de las autoridades del Hospital Universitario de Guayaquil y de la Universidad de Guayaquil para su ejecución, el cual será supervisado por un tutor.

\section{Criterios de inclusión}

Todos los Internos de Medicina de la Universidad de Guayaquil y los Médicos Residentes que laboren en el Área de Emergencia del Hospital Universitario durante el periodo de septiembre a diciembre de 2015,sin restricción de edad o género o etnia.

\section{Criterios de exclusión}

Licenciadas (os) y Auxiliares de Enfermería que laboren en el área de emergencia y los Internos de Medicina y Médicos Residentes que se nieguen a realizar la encuesta.

Operacionalizacion de los instrumentos de investigación

Para la siguiente investigación se utilizaron encuestas, conformadas por datos demográficos, factores de riesgo, y uso del cuestionario de Maslach Burnout Inventory el mismo 
Síndrome de desgaste profesional en médicos residentes e internos de medicina del área de emergencia del hospital universitario periodo septiembre 2015 a diciembre 2015

Vol. 3, núm. 3 Esp., (2019)

Charles David Altamirano Olvera; Elizabeth Katiuska Villegas Guerrero; Sacoto Carranza Henrry Willian; González Plúa Carmen Leonor

que se aplicó a médicos residentes del Hospital Universitario e internos de la universidad de Guayaquil, se recogió información vía online (correo electrónico, WhatsApp, facebook).

\section{Resultados.}

Este estudio fue realizado a los Internos de Medicina de la Universidad de Guayaquil y Médicos Residentes del área de emergencia del Hospital universitario de Guayaquil. Los resultados están representado por medio de la utilización de estadística descriptiva a través de tablas de frecuencias y gráficos ilustrativos realizados en Microsoft Excel. 
Síndrome de desgaste profesional en médicos residentes e internos de medicina del área de emergencia del hospital universitario periodo septiembre

2015 a diciembre 2015

Vol. 3, núm. 3 Esp., (2019)

Charles David Altamirano Olvera; Elizabeth Katiuska Villegas Guerrero; Sacoto Carranza Henrry Willian; González Plúa Carmen Leonor

Tabla $\mathbf{N}^{\circ}$ 1. Distribución de frecuencias de las variables independientes de los internos de medicina de la universidad de Guayaquil y médicos residentes del área de emergencia del hospital universitario de Guayaquil.

\begin{tabular}{|c|c|c|c|}
\hline \multirow[t]{2}{*}{ VARIABLES } & \multicolumn{2}{|c|}{ FRECUENCIA } & \multirow{2}{*}{\begin{tabular}{|l|}
$\%$ \\
70
\end{tabular}} \\
\hline & $24-29$ & 73 & \\
\hline \multirow[t]{2}{*}{ Edad } & \begin{tabular}{|l|}
$30-35$ \\
\end{tabular} & 20 & 19 \\
\hline & $36-41$ & 11 & 11 \\
\hline \multirow{3}{*}{ Género } & Masculino & 46 & 44 \\
\hline & Femenino & 58 & 56 \\
\hline & GLBTI & 0 & 0 \\
\hline \multirow{5}{*}{ Estado civil } & Soltero & 67 & 64 \\
\hline & Unión libre & 8 & 8 \\
\hline & Casado & 28 & 27 \\
\hline & Divorciado & 1 & 1 \\
\hline & Viudo & 0 & 0 \\
\hline \multirow{2}{*}{$\begin{array}{c}\text { Relación } \\
\text { sentimental }\end{array}$} & $\mathrm{Si}$ & 62 & 60 \\
\hline & No & 42 & 40 \\
\hline \multirow{3}{*}{ Convivencia } & Padres & 64 & 62 \\
\hline & Pareja & 26 & 25 \\
\hline & Solo & 14 & 13 \\
\hline \multirow{2}{*}{ Hijos } & $\mathrm{Si}$ & 56 & 54 \\
\hline & No & 48 & 46 \\
\hline
\end{tabular}


Síndrome de desgaste profesional en médicos residentes e internos de medicina del área de emergencia del hospital universitario periodo septiembre 2015 a diciembre 2015

Vol. 3, núm. 3 Esp., (2019)

Charles David Altamirano Olvera; Elizabeth Katiuska Villegas Guerrero; Sacoto Carranza Henrry Willian; González Plúa Carmen Leonor

\begin{tabular}{|c|c|c|c|}
\hline VARIABLES & \multicolumn{2}{|c|}{ FRECUENCIA } & $\%$ \\
\hline \multirow{2}{*}{$\begin{array}{c}\text { Consumo de } \\
\text { alcohol }\end{array}$} & $\mathrm{Si}$ & 66 & 63 \\
\hline & $\mathrm{No}$ & 38 & 37 \\
\hline \multirow{2}{*}{$\begin{array}{c}\text { Actividad } \\
\text { extracurricular }\end{array}$} & $\mathrm{Si}$ & 36 & 35 \\
\hline & No & 68 & 65 \\
\hline \multirow{2}{*}{$\begin{array}{c}\text { Descanso durante } \\
\text { la guardia }\end{array}$} & SI & 74 & 71 \\
\hline & $\mathrm{NO}$ & 30 & 29 \\
\hline \multirow{2}{*}{$\begin{array}{l}\text { Consumo de } \\
\text { cigarrillo }\end{array}$} & $\mathrm{Si}$ & 62 & 60 \\
\hline & No & 38 & 37 \\
\hline \multirow{3}{*}{ Remuneración } & $<\$ 500$ & 0 & 0 \\
\hline & $\$ 500-\$ 700$ & 72 & 69 \\
\hline & $>\$ 700$ & 32 & 31 \\
\hline \multirow{2}{*}{$\begin{array}{c}\text { Descanso post- } \\
\text { guardia }\end{array}$} & $\mathrm{Si}$ & 86 & 83 \\
\hline & No & 18 & 17 \\
\hline \multirow{3}{*}{$\begin{array}{c}\text { Relación con el } \\
\text { equipo }\end{array}$} & Buena & 88 & 85 \\
\hline & Regular & 12 & 12 \\
\hline & Mala & 4 & 4 \\
\hline \multirow{3}{*}{$\begin{array}{c}\text { Área de } \\
\text { emergencia }\end{array}$} & Adultos & 48 & 46 \\
\hline & Pediatría & 28 & 27 \\
\hline & Ginecología & 28 & 27 \\
\hline
\end{tabular}

Fuente: Cuestionario de datos demográficos y factores de riesgo

Elaborado por: Charles David Altamirano Olvera

Resultado: En la tabla 1 se establece el porcentaje de frecuencia de las variables independientes en el universo (104) teniendo en cuenta que el $46 \%$ en estudio son de Emergencia Adultos, el 27\% de Emergencia Pediátrica y el 27\% de Emergencia Ginecológica. 
Síndrome de desgaste profesional en médicos residentes e internos de medicina del área de emergencia del hospital universitario periodo septiembre

2015 a diciembre 2015

Vol. 3, núm. 3 Esp., (2019)

Charles David Altamirano Olvera; Elizabeth Katiuska Villegas Guerrero; Sacoto Carranza Henrry Willian; González Plúa Carmen Leonor

Tabla $\mathbf{N}^{\circ}$ 2. Distribución de frecuencias por dimensiones del síndrome de desgaste profesional en los internos de medicina de la universidad de Guayaquil en el área de emergencia del hospital universitario.

\begin{tabular}{|c|l|r|r|}
\hline VARIABLE & \multicolumn{2}{|c|}{ FRECUENCIA } & \% \\
\hline \multirow{3}{*}{ Cansancio emocional } & ALTO & 49 & 68 \\
\cline { 2 - 4 } & MEDIO & 10 & 14 \\
\cline { 2 - 4 } & BAJO & 13 & 18 \\
\hline \multirow{3}{*}{ Despersonalización } & ALTO & 42 & 58 \\
\cline { 2 - 4 } & MEDIO & 11 & 15 \\
\cline { 2 - 4 } & BAJO & 19 & 26 \\
\hline \multirow{3}{*}{ Realización personal } & ALTO & 12 & 17 \\
\cline { 2 - 4 } & MEDIO & 18 & 25 \\
\cline { 2 - 5 } & BAJO & 42 & 58 \\
\hline
\end{tabular}

Fuente: Encuesta de Maslach Burnout Inventory

Elaborado por: Charles David Altamirano Olvera

Resultado: En la tabla 2 se muestra que el 68\% de los Internos de Medicina de la Universidad de Guayaquil encuestados presentaron altos niveles de cansancio emocional, el 58\% despersonalización y el 17\% falta de realización personal. 
Síndrome de desgaste profesional en médicos residentes e internos de medicina del área de emergencia del hospital universitario periodo septiembre 2015 a diciembre 2015

Vol. 3, núm. 3 Esp., (2019)

Charles David Altamirano Olvera; Elizabeth Katiuska Villegas Guerrero; Sacoto Carranza Henrry Willian; González Plúa Carmen Leonor

Tabla $\mathbf{N}^{\circ}$ 3. Distribución de frecuencias por dimensiones del síndrome de desgaste profesional en

los médicos residentes del área de emergencia del hospital universitario de Guayaquil.

\begin{tabular}{|c|l|r|r|}
\hline \multicolumn{1}{|c|}{ VARIABLE } & \multicolumn{2}{|c|}{ FRECUENCIA } & $\%$ \\
\hline \multirow{3}{*}{ Cansancio emocional } & ALTO & 18 & 56 \\
\cline { 2 - 5 } & MEDIO & 8 & 25 \\
\cline { 2 - 5 } & BAJO & 6 & 19 \\
\hline \multirow{3}{*}{ Despersonalización } & ALTO & 14 & 44 \\
\cline { 2 - 5 } & MEDIO & 10 & 31 \\
\cline { 2 - 5 } & BAJO & 8 & 25 \\
\hline \multirow{3}{*}{ Realización personal } & ALTO & 7 & 22 \\
\cline { 2 - 5 } & MEDIO & 10 & 31 \\
\cline { 2 - 5 } & BAJO & 15 & 47 \\
\hline
\end{tabular}

Fuente: Encuesta de Maslach Burnout Inventory

Realizada por: Charles David Altamirano Olvera

Resultado: En la tabla 3 se muestra que el 56\% de los Médicos Residentes encuestados presentaron altos niveles de cansancio emocional, el $44 \%$ despersonalización y el $22 \%$ falta de realización personal. 
Síndrome de desgaste profesional en médicos residentes e internos de medicina del área de emergencia del hospital universitario periodo septiembre

2015 a diciembre 2015

Vol. 3, núm. 3 Esp., (2019)

Charles David Altamirano Olvera; Elizabeth Katiuska Villegas Guerrero; Sacoto Carranza Henrry Willian; González Plúa Carmen Leonor

Tabla 4. Distribución de frecuencias por dimensiones del síndrome de desgaste profesional en los internos de medicina de la universidad de Guayaquil y médicos residentes del área de emergencia del hospital universitario de Guayaquil.

\begin{tabular}{|c|l|r|r|}
\hline \multicolumn{1}{|c|}{ VARIABLE } & \multicolumn{2}{|c|}{ FRECUENCIA } & \% \\
\hline \multirow{3}{*}{ Cansancio emocional } & ALTO & 67 & 64 \\
\cline { 2 - 4 } & MEDIO & 18 & 17 \\
\cline { 2 - 4 } & BAJO & 19 & 18 \\
\hline \multirow{3}{*}{ Despers onalización } & ALTO & 56 & 54 \\
\cline { 2 - 4 } & MEDIO & 21 & 20 \\
\cline { 2 - 4 } & BAJO & 27 & 26 \\
\hline \multirow{3}{*}{ Realización pers onal } & ALTO & 19 & 18 \\
\cline { 2 - 4 } & MEDIO & 28 & 27 \\
\cline { 2 - 4 } & BAJO & 57 & 55 \\
\hline SDP & $\uparrow C E \uparrow D P \downarrow R P$ & 17 & 16 \\
\hline
\end{tabular}

Fuente: Encuesta de Maslach Burnout Inventory

Realizada por: Charles David Altamirano Olvera

Resultado: En la tabla 4 se muestra en términos generales que de los 104 encuestados que el 64\% presentaron altos niveles de cansancio emocional, el 54\% despersonalización y el 18\% falta de realización personal. 


\section{Síndrome de desgaste profesional en médicos residentes e internos de medicina del área de emergencia del hospital universitario periodo septiembre 2015 a diciembre 2015}

Vol. 3, núm. 3 Esp., (2019)

Charles David Altamirano Olvera; Elizabeth Katiuska Villegas Guerrero; Sacoto Carranza Henrry Willian; González Plúa Carmen Leonor

Tabla 5. Síndrome de desgaste profesional en internos de medicina de la universidad de Guayaquil y médicos residentes del área de emergencia del hospital universitario de Guayaquil.

\begin{tabular}{|l|r|r|r|r|}
\hline ÁREA & INTERNOS & RESIDENTES & \multicolumn{1}{|c|}{ SDP } & \multicolumn{1}{|c|}{$\%$} \\
\hline EMERGENCIA DE ADULTOS & 12 & 2 & 14 & 13 \\
\hline EMERGENCIA PEDIATRICA & 0 & 0 & 0 & 0 \\
\hline EMERGENCIA DE GINECOLOGÍA & 3 & 0 & 3 & 3 \\
\hline NO CUMPLIERON CRITERIO & 57 & 30 & 87 & 84 \\
\hline
\end{tabular}

Fuente: Encuesta de Maslach Burnout Inventory

Realizada por: Charles David Altamirano Olvera

Resultado: En la tabla 5 podemos observar con más detalle los afectados por el Síndrome de Desgaste Profesional por Área de Emergencia del Hospital Universitario de Guayaquil entre Internos de Medicina de la Universidad de Guayaquil y Médicos Residentes teniendo como resultado el 13\% en Emergencia Adultos y 3\% en Emergencia Ginecológica sumando un total del $16 \%$, se puede observar que el $84 \%$ no cumplieron criterio y que Emergencia Pediátrica no se vio afectada.

En términos generales que de los 104 encuestados que el 64\% presentaron altos niveles de cansancio emocional, (en relación con el 68\% de internos y el 56\% de médicos residentes) el 54\% despersonalización ( en relación al 58\% de los internos y el 44\% de los médicos residentes) y el $18 \%$ falta de realización personal (En relación al 17\% de los internos y el 22\% de los médicos residentes) cumpliendo así el criterio de puntuaciones altas en las tres dimensiones $\uparrow \mathrm{CE} \uparrow \mathrm{DP} \downarrow \mathrm{RP}$ lo que nos lleva a un $16 \%$ de nuestra muestra de estudio que presentó el síndrome, dado que el Los 
Síndrome de desgaste profesional en médicos residentes e internos de medicina del área de emergencia del hospital universitario periodo septiembre

2015 a diciembre 2015

Vol. 3, núm. 3 Esp., (2019)

Charles David Altamirano Olvera; Elizabeth Katiuska Villegas Guerrero; Sacoto Carranza Henrry Willian; González Plúa Carmen Leonor

profesionales de la salud son uno de los colectivos que sufre altos niveles de estrés laboral, debido a la responsabilidad de la salud de los pacientes, su contacto diario con la muerte y el dolor, los turnos de 24 horas más horas asistenciales, la carga laboral, la presión social influyen en la aparición del síndrome.

La edad media de presentación fue de 26.6 años, la mediana es de 26 años, presentación modal de 26 años y una desviación estándar de 2.44 años. 
Síndrome de desgaste profesional en médicos residentes e internos de medicina del área de emergencia del hospital universitario periodo septiembre 2015 a diciembre 2015

Vol. 3, núm. 3 Esp., (2019)

Charles David Altamirano Olvera; Elizabeth Katiuska Villegas Guerrero; Sacoto Carranza Henrry Willian; González Plúa Carmen Leonor

Tabla 6. Variables y dimensiones del síndrome de desgaste profesional en internos de medicina de la universidad de Guayaquil y médicos residentes del área de emergencia del hospital universitario de Guayaquil.

\begin{tabular}{|c|c|c|c|c|c|c|c|}
\hline \multicolumn{2}{|c|}{ VARIABLES } & \multicolumn{2}{|c|}{$\mathbf{C E}$} & \multicolumn{2}{|c|}{ DP } & \multicolumn{2}{|c|}{$\mathbf{R P}$} \\
\hline & & \multirow{2}{*}{\begin{tabular}{|l|l} 
F & 0 \\
56 &
\end{tabular}} & \multirow{2}{*}{\begin{tabular}{|l|l}
$\%$ \\
77
\end{tabular}} & \multirow{2}{*}{\begin{tabular}{|l|l} 
F & 0 \\
50
\end{tabular}} & \multirow{2}{*}{\begin{tabular}{|l|}
$\%$ \\
68 \\
\end{tabular}} & & \multirow{2}{*}{\begin{tabular}{|l|}
$\%$ \\
21 \\
\end{tabular}} \\
\hline \multirow{3}{*}{ Edad } & $24-29$ & & & & & & \\
\hline & $30-35$ & 7 & 35 & 5 & 25 & 2 & 10 \\
\hline & $36-41$ & 4 & 36 & 1 & 9 & 2 & 18 \\
\hline \multirow{3}{*}{ Género } & Masculino & 20 & 43 & 18 & 39 & 16 & 35 \\
\hline & Femenino & 47 & 81 & 38 & 66 & 3 & 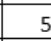 \\
\hline & GLBTI & 0 & 0 & 0 & 0 & 0 & 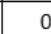 \\
\hline \multirow{5}{*}{ Estado civil } & Soltero & 45 & 67 & 32 & 48 & 10 & 15 \\
\hline & Unión libre & 5 & 63 & 6 & 75 & 5 & 63 \\
\hline & \begin{tabular}{|l|} 
Casado \\
\end{tabular} & 17 & 61 & 18 & 64 & 4 & 14 \\
\hline & Divorciado & 0 & 0 & 0 & 0 & 0 & 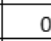 \\
\hline & Vrudo & 0 & 0 & 0 & 0 & 0 & 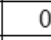 \\
\hline \multirow{2}{*}{$\begin{array}{c}\text { Relación } \\
\text { sentimental }\end{array}$} & $\mathrm{Si}$ & 29 & 47 & 36 & 58 & 11 & 18 \\
\hline & No & 38 & 90 & 20 & 48 & 8 & 19 \\
\hline \multirow{3}{*}{ Convivencia } & Padres & 42 & 66 & 40 & 63 & 8 & 13 \\
\hline & Pareja & 18 & 69 & 8 & 31 & 7 & 27 \\
\hline & Solo & 7 & 50 & 8 & 57 & 4 & 29 \\
\hline \multirow{2}{*}{ Hijos } & $\mathrm{Si}$ & 48 & 86 & 42 & 75 & 12 & 21 \\
\hline & No & 19 & 40 & 14 & 29 & 7 & 15 \\
\hline \multirow{2}{*}{$\begin{array}{c}\text { Consumo de } \\
\text { alcohol }\end{array}$} & $\mathrm{Si}$ & 45 & 68 & 38 & 58 & 13 & 20 \\
\hline & No & 22 & 58 & 18 & 47 & 6 & 16 \\
\hline \multirow{2}{*}{\begin{tabular}{|c} 
Actividad \\
extracurricular
\end{tabular}} & $\mathrm{Si}$ & 30 & 83 & 15 & 42 & 14 & 39 \\
\hline & No & 37 & 54 & 41 & 60 & 5 & \\
\hline \multirow{2}{*}{\begin{tabular}{|c|}
$\begin{array}{c}\text { Descanso dur ante } \\
\text { la gyar dia }\end{array}$ \\
\end{tabular}} & SI & 41 & 55 & 31 & 42 & 4 & 5 \\
\hline & NO & 26 & 87 & 25 & 83 & 15 & 50 \\
\hline \multirow{2}{*}{$\begin{array}{l}\text { Consumo de } \\
\text { ciganillo }\end{array}$} & $\mathrm{Si}$ & 39 & 63 & 29 & 47 & 11 & 18 \\
\hline & No & 28 & 74 & 27 & 71 & 8 & 21 \\
\hline \multirow{3}{*}{ Remuneración } & $<\$ 500$ & 0 & 0 & 0 & 0 & 0 & 0 \\
\hline & $\$ 500-\$ 70$ & 51 & 71 & 43 & 60 & 17 & 24 \\
\hline & $>\$ 700$ & 16 & 50 & 13 & 41 & 2 & 6 \\
\hline \multirow{2}{*}{\begin{tabular}{|c}
$\begin{array}{c}\text { Descanso post- } \\
\text { guardia }\end{array}$ \\
\end{tabular}} & $\mathrm{Si}$ & 50 & 58 & 39 & 45 & 10 & 12 \\
\hline & No & 17 & 94 & 17 & 94 & 9 & 50 \\
\hline \multirow{3}{*}{$\begin{array}{c}\text { Relación con e } \\
\text { equipo }\end{array}$} & Buema & 56 & 64 & 46 & 52 & 9 & 10 \\
\hline & Regular & 8 & 67 & 7 & 58 & 6 & 50 \\
\hline & Mala & 3 & 75 & 3 & 75 & 4 & 100 \\
\hline \multirow{3}{*}{$\begin{array}{l}\text { Área de } \\
\text { emergencia }\end{array}$} & Adultos & 42 & 88 & 37 & 77 & 11 & 23 \\
\hline & Pediatría & 8 & 29 & 6 & 21 & 2 & 7 \\
\hline & Ginecologia & 17 & 61 & 13 & 46 & 6 & 21 \\
\hline
\end{tabular}

Fuente: Cuestionario de datos demográficos y factores de riesgo

Elaborado por: Charles David Altamirano Olvera 


\section{Síndrome de desgaste profesional en médicos residentes e internos de medicina del área de emergencia del hospital universitario periodo septiembre}

2015 a diciembre 2015

Vol. 3, núm. 3 Esp., (2019)

Charles David Altamirano Olvera; Elizabeth Katiuska Villegas Guerrero; Sacoto Carranza Henrry Willian; González Plúa Carmen Leonor

\section{Conclusiones.}

El 16\% del universo cumplió el criterio de puntuaciones altas en las tres dimensiones $\uparrow \mathrm{CE} \uparrow \mathrm{DP} \downarrow \mathrm{RP}$ lo que nos lleva a un $16 \%$ de nuestra muestra de estudio que presentó el síndrome de desgaste profesional. A su vez la edad media de presentación del síndrome de desgaste profesional fue de 26.6 años, con una desviación estándar de 2.44 años.

Numerosos estudios refieren que el grupo etario donde mayormente se presenta este síndrome es en personas jóvenes entre los 30 y 40 años o menores, ya que en edades avanzadas se adquiere mayor seguridad en el desarrollo de las actividades y se hace menos vulnerable al estrés laboral.

El grupo más vulnerable fue la mujer en un $70 \%$ investigaciones realizadas lo atribuyen a dos factores fundamentales que son la sobrecarga de trabajo profesional y la tarea familiar, así como la elección de determinadas especialidades profesionales que prolongarían el rol de mujer.

Respecto al estado civil, estudios demuestran que el síndrome de desgaste profesional está presente en profesionales que no tienen pareja estable, en comparación con las que están casadas o conviven con su parejas, en este mismo orden la existencia o no de hijos, hace que estas personas puedan ser más resistentes al síndrome, la implicación con la familia hace que tengan mayor capacidad para afrontar problemas personales y conflictos emocionales y que sean más realistas con el apoyo familiar. Aunque el Síndrome se ha asociado más con personas que no tienen pareja estable en mi estudio se presentó de manera equilibrada $35 \%$ no vivían con pareja $33 \%$ vivían con sus padres y el $32 \%$ restante vivían con pareja. 


\section{Síndrome de desgaste profesional en médicos residentes e internos de medicina del área de emergencia del hospital universitario periodo septiembre 2015 a diciembre 2015}

Vol. 3, núm. 3 Esp., (2019)

Charles David Altamirano Olvera; Elizabeth Katiuska Villegas Guerrero; Sacoto Carranza Henrry Willian; González Plúa Carmen Leonor

La deprivación del sueño y su efecto en el desempeño laboral y académico ha sido estudiado y recientemente sujeto a regulaciones, está comprobado que la deprivación crónica del sueño modifica la concentración y altera la capacidad de tomar decisiones que como resultado se incrementan los errores con consecuencias fatales.

Algo que podríamos destacar en este estudio es que se da con mayor frecuencia en la emergencia de adultos en un $13 \%$, debido al no descanso durante la guardia que se destacó en un 90\%, seguido de emergencia ginecológica de un $3 \%$ y no viéndose afectada emergencia pediátrica.

Es importante mencionar que el $21 \%$ de los Internos de Medicina en estudio fueron afectados en comparación con el $6 \%$ de Médicos Residentes afectados llegando a no cumplir criterio el $84 \%$ en estudio pero presentando algunas de las dimensiones de desgaste profesional que numerosos estudios mencionan que la fluidez de pacientes, espacio reducido para la atención, mayor contacto con la muerte y el dolor de los usuarios hace que se desarrolle más el síndrome.

\section{Bibliografía.}

Ewart, Q., Marcus, L., Gaba, M., \& Brandnen, R. (2004). The critical care medicine crisis: a call for federal action a white paper from the critical care professional societies. Chest, 125(4).

Galván, M., Vassallo, J., Rodríguez, S., \& Otero, P. (2014). Síndrome de desgaste profesional (burnout) en médicos de unidades de cuidados intensivos pediátricos en la Argentina. Revista de la Sociedad Boliviana de Pediatría, 53(1), 29-36.

Lopez León, J., Rodríguez Moctensuma, J., \& López Carbona, M. L. (2007). Desgaste profesional en médicos familaires y su asociación con factores sociodemográficos y laborales. Rev Med Inst Mex Seguro Soc. , 14.

OMS. (12 de febrero de 2016). Organización Mundial de la salud. Obtenido de http://www.who.int/topics/es/ 
Síndrome de desgaste profesional en médicos residentes e internos de medicina del área de emergencia del hospital universitario periodo septiembre 2015 a diciembre 2015

Vol. 3, núm. 3 Esp., (2019)

Charles David Altamirano Olvera; Elizabeth Katiuska Villegas Guerrero; Sacoto Carranza Henrry Willian; González Plúa Carmen Leonor

Quevedo Bastias, K. (2006). Síndrome de Burnout y calidad de atención en el área de emergencia del Hospital Regional" Dr. Teodoro Maldonado Carbo". MEDICINA, 18.

Sos Tena, P., Sobrequés Soriano, J., \& Segura Bernal, J. (2002). Desgaste profesional en los médicos de Atención Primaria de Barcelona. Desgaste profesional en los médicos de Atención Primaria de Barcelona, 12(10), 613-619.

$$
\text { (c) }(1)(2)(2)
$$

RECONOCIMIENTO-NOCOMERCIAL-COMPARTIRIGUAL

CC BY-NC-SA

ESTA LICENCIA PERMITE A OTROS ENTREMEZCLAR, AJUSTAR Y CONSTRUIR A PARTIR DE SU OBRA CON FINES NO COMERCIALES, SIEMPRE Y CUANDO LE RECONOZCAN LA AUTORÍA Y SUS NUEVAS CREACIONES ESTÉN BAJO UNA LICENCIA CON LOS MISMOS TÉRMINOS. 